\title{
Markers of Metastatic Potential in Prostate Cancer using Magnetic Resonance Spectroscopy
}

Khushali Kotedia ${ }^{1,2}$, Calvin Ryan ${ }^{3}$, Hanna M Romanska ${ }^{1}$, El-Nasir Lalani ${ }^{1}$, I Jane Cox ${ }^{2}$

${ }^{1}$ Department of Histopathology, Division of Investigative Sciences, ${ }^{2}$ Imaging Sciences

Department, Clinical Sciences Centre, ${ }^{3}$ Postgraduate Education, Division of Surgery,

Faculty of Medicine, Hammersmith Campus, Imperial College London

\section{Introduction.}

A major focus of translation research is to apply emerging technologies towards defining the malignant phenotype and to establish whether the malignant phenotype can differentiate normal from malignant. One such technique is magnetic resonance spectroscopy (MRS), a versatile noninvasive investigative technique based on the measurement of magnetic signals from nuclei responding to radiofrequency pulses. Initial clinical results of MRS studies of prostate cancer (PCa) have demonstrated a significant reduction of citrate and an elevation of choline containing compounds (Cho) in $\mathrm{PCa}$ compared with normal prostatic peripheral zone tissue and benign prostatic hypertrophy (Kurhanewicz et al. JMRI 2002; 16: 451-463). Studies of surgical prostate cancer specimens have demonstrated that compounds involved in phosphotidylcholine and phosphotidylethanolamine synthesis and hydrolysis contribute to the MR spectrum. Therefore, as in other human cancers, the elevation of the Cho peak is associated with changes in cell membrane synthesis and degradation that occur with the evolution of cancer. However the significance and specificity of these changes remains unclear. In this study intact prostatic cancer cell lines were profiled using ${ }^{1} \mathrm{H}$ magic angle spinning (MAS) MRS in order to establish whether MRS profiles could complement phenotypic characteristics of neoplastic cells.

Materials and Methods. The human prostate derived tumourogenic cell lines studied are summarised in the Table:

\begin{tabular}{|l|l|l|l|}
\hline Cell line & Metastatic Potential & AR protein & Origin \\
\hline DU145 & High $(>90 \%)$ & Negative & Brain metastasis \\
\hline PC3 & High $(>90 \%)$ & Negative & Vertebral metastasis \\
\hline LNCaP & Low $(<10 \%)$ & Positive & Lymph node metastasis \\
\hline
\end{tabular}

All cell lines were grown in RPMI, supplemented with $10 \%$ fetal bovine serum (FBS). Cells were phenotyped immunocytochemically as previously described to ensure purity and obtain a partial fingerprint at the start of the studies (Mitchell et al. BJU Int 2000; 85: 932-944). Between $1 \times 10^{6}$ $5 \times 10^{6}$ cells were placed in the 20_I sample holder for MAS. MAS MRS is a method where line broadening due to anisotropic interactions in solid materials is reduced by spinning the sample about its own axis at $4000 \mathrm{~Hz}$ at a $54.7^{\circ}$ angle to the static magnetic field (Weybright et al. MRM 1998; 39: 337-344). ${ }^{1} \mathrm{H}$ MAS MR spectra were acquired using a Doty CP/MAS probe interfaced with a JEOL Eclipse $500+$ spectrometer (JEOL, UK) at a temperature of $25^{\circ} \mathrm{C}$. Spectra were acquired with water pre-saturation using pulse-collect techniques and analysed using the fitting program AMARES.

Results Resonances assigned to choline-containing compounds (choline, phosphocholine, glycerophosphocholine and phosphotidylcholine) and to lipids $\left(-\mathrm{CH}_{3}\right.$ at $0.9 \mathrm{ppm},-\mathrm{CH}_{2}$ at $1.3 \mathrm{ppm}$ and $2 \mathrm{ppm}$ ) were observed in all spectra. The peak area ratio of (total Cho)/(total lipid) was significantly different between the cell lines (PC3: 0.206 \pm 0.008 , DU145: 0.133 \pm 0.005 ; LNCaP: $0.100 \pm 0.002), p<0.001$, ANOVA.

Conclusions. The MR spectral profile of human prostate derived tumourogenic cell lines is characteristic of the cell line phenotype, indicating that it may be possible to identify MR markers of metastatic potential in prostate cancer. 